\title{
True Colors: A Century of Synthetic Dyes
}

For more than 5,000 years, mankind has brought color into the world by extracting various pigments and using them to dye textiles, leather, paper, wood, and many other things. Up until the last century, though, all such pigments were obtained either directly or indirectly from natural sources.

Most natural dyes come from plant parts-the berries, bark, leaves, flowers, or roots. The madder plant native to Asia and Europe provided bright red color for linen and silk. The crocus plant gave a yellow saffron dye. The little Indigofera Tinctoria bush, mainly from India, supplied a muchprized dark-blue color. The logwood tree from Mexico, Central America, and the West Indies yielded black and brown dyes. The henna shrub of North Africa and the Middle East gave a reddish-brown color, chiefly used to dye leather.

Some dyes were derived from animal sources. Best known is carmine, a bright red extracted from the dried bodies of the female cochineal insect of Mexico and Central America. "Tyrian purple" of ancient times, reserved for royalty because of its scarcity, was obtained only from some Mediterranean shellfish.

Unfortunately, most natural dye substances do not bond firmly to textiles-they fade with sunlight or wash out with water.

Not until 1856 was the first synthetic dye discovered. English chemist William $\mathrm{H}$. Perkin stumbled on the pale purple color, mauve, while searching for something completely unrelated. In 1855 Perkin, the son of a carpenter who did not approve of young William's interest in chemistry, became the assistant of the German chemist A.W. von Hofmann. Hofmann had speculated aloud on the possibility of synthesizing quinine in the laboratory. Quinine, the valuable alkaloid used to fight against malaria, was obtained from the bark of the tropical cinchona tree. If it could instead be made from cheap coal-tar chemicals, it would be a boon to medicine and amass a fortune for the inventor of the process because it would eliminate European dependence on distant imports.

Excited by the idea, Perkin went home during his Easter vacation in 1856 to try on his own. He was enthusiastic-and only 18 at the time-but his attempt was not successful for many reasons. The chemical structure of quinine was not known at the time, and even if it had been the primitive synthetic methods available were not sufficient to the task.

But in one of his attempts, Perkin mixed the coal-tar derivative aniline with potassium dichromate. Before pouring out the failed mixture, he noticed a purplish tint to the liquid. By some instinct, he decided to add alcohol to the compound, which dissolved out a beautiful purple precipitate.

Perkin immediately wondered if the substance might be used as a dye. Earlier, he and Arthur $\mathrm{H}$. Church had patented a process for obtaining "nitrosonaphthalene" by reducing dinitrobenzene and dinitronaphthalene, which turned out to be the first of the azo-dyes used in later manufacturing. Perkin sent a sample of his new purple compound to a dyeing company in Perth, Scotland, and they responded quickly that it dyed silk beautifully. "If your discovery does not make the goods too expensive," they wrote, "it is decidedly one of the most valuable that has come out for a long time."

Perkin immediately went about obtaining a patent for his process, though he had some difficulty because it was questioned whether an 18-year-old boy could hold a patent of his own.

\section{William Perkin discov- ered the first synthetic dye while trying to synthesize quinine.}

Despite the protests of his mentor Hofmann, Perkin left school and set out in June 1857 to form a dye factory of his own at Greenford Green. Perkin's father, who had previously frowned on his son's interest in chemistry, now gave his wholehearted support to the venture, contributing his life savings as capital.

However, problems arose. Perkin had used aniline as his starting material for the "aniline purple" or "Tyrian purple" dye, named after the expensive shellfishderived dye used for ancient royalty-but facilities and techniques for manufacturing large amounts of aniline had not yet been established. Perkin had to do the manufacturing himself, buying benzene as a raw material and making his own strong nitric acid (which was also not available) for the process to convert benzene into aniline. At several other steps along the way, Perkin had to develop his own techniques or design his own equipment. Even with these stumbling blocks, Perkin placed his "aniline purple" on the market within six months!

The English dyers showed some reluctance to use the new dye, despite Perkin's experience with the Scottish company; but French dyers embraced the aniline purple with enormous enthusiasm. They called the color "mauve" after their word for the madder plant, also used in dyeing. The dye became so popular in France that some historians refer to this period as the "Mauve Decade."

At age 23, and somewhat to his surprise, Perkin had become the world authority on dyes. By the time he turned 35, he was independently wealthy and, not wanting to compete with the growing German dye industry, he sold his factory and spent the rest of his life pursuing his own chemical research. He received the Royal Society's Davy Medal in 1889, and was knighted in 1906, a year before he died.

The discovery of one synthetic dye sparked a great deal of interest in finding other colors, which led to many new developments in organic chemistry. Perkin's own son, William Perkin Jr., helped develop synthetic indigo, one of the most important commercial dyes.

Natural indigo had been known since the time of ancient Egypt, Greece, and Rome. The indigo shrub is native to Asia and India, 4 to 6 feet high, and bears small yellow flowers. The name "indigo" is a Spanish form of the English word "India."

Indigo dye is produced indirectly from the indigo plant by oxidation of a glucoside extracted from the herbage. Freshly harvested plants are steeped in water and allowed to ferment for 10-15 hours. The resulting yellow liquid is drained off into vats equipped with paddle wheels that splash the liquid into the air. The droplets oxidize, and a dark-blue sediment of indigo settles to the bottoms of the vats. The sediment is collected, washed, dried, and pressed into small cubes.

Indigo was used for dyeing cotton, wool, and other fabrics, as well as for a pigment in paints and inks. Indigo was extremely 
important because of its deep color and its permanence. Many of the world's navies used uniforms colored with the deep-blue indigo, "navy blue," because only indigo dye could withstand the harsh salt water and pounding sunlight endured by sailors.

Cultivation of indigo plants stretched from the East Indies to the New World, and the first American plantation began in South Carolina in 1742. During the British occupation of India, indigo became a major export. In 1897 British India had 1,750,000 acres of land planted with indigo, and exported 8,500 tons in that year alone. However, with the discovery of synthetic indigo dye, and after considerable economic upheaval in India, the cultivation of indigo plants has practically ceased there.

German chemist Adolf von Baeyer, working with William Perkin Jr., announced that he had created synthetic indigo in 1878 and he obtained a patent, although his process was difficult and not useful for manufacturing purposes. Nevertheless, in 1880 two large chemical and dye organizations, the Badische Anilinund Soda-Fabrik and the Hoechst Farbewerke, acquired his patents. Three years later Baeyer determined the exact chemical structure of indigo $\left(\mathrm{C}_{10} \mathrm{H}_{10} \mathrm{~N}_{2} \mathrm{O}_{2}\right)$, which launched a race among the German dyestuff companies to find a commercially viable process to synthesize the dye. It took them 15 years to succeed.

The dye companies had expended large sums of money on their research, but Baeyer declined to participate in the venture to commencialize his discovery. Resulting ill-feelings from the dye manufacturers caused him to discontinue his work on the subject and concentrate on other aspects of organic chemistry. Nevertheless, Baeyer won the Nobel Prize in chemistry in 1905 "in recognition of his services in the development of organic chemistry and the chemical industry through his work on organic dyes and hydro-aromatic combinations."

Because of Baeyer's impetus, Germany controlled most of the world's dye production until World War I, when the Germans cut off their supply of dyes. This allowed the U.S. dye industry to grow and fill the void.

With the abundance of new polymers and synthetic fibers since 1940, chemists have been forced to continue developing appropriate dyes and bonding agents for them. At present, the number of different dyes used by U.S. industries has grown to more than 8,000 .
Anatech's R.F. Planar Magnetron and D.C. Sputtering System

\section{THIN FILM DEPOSITION SYSTEM}

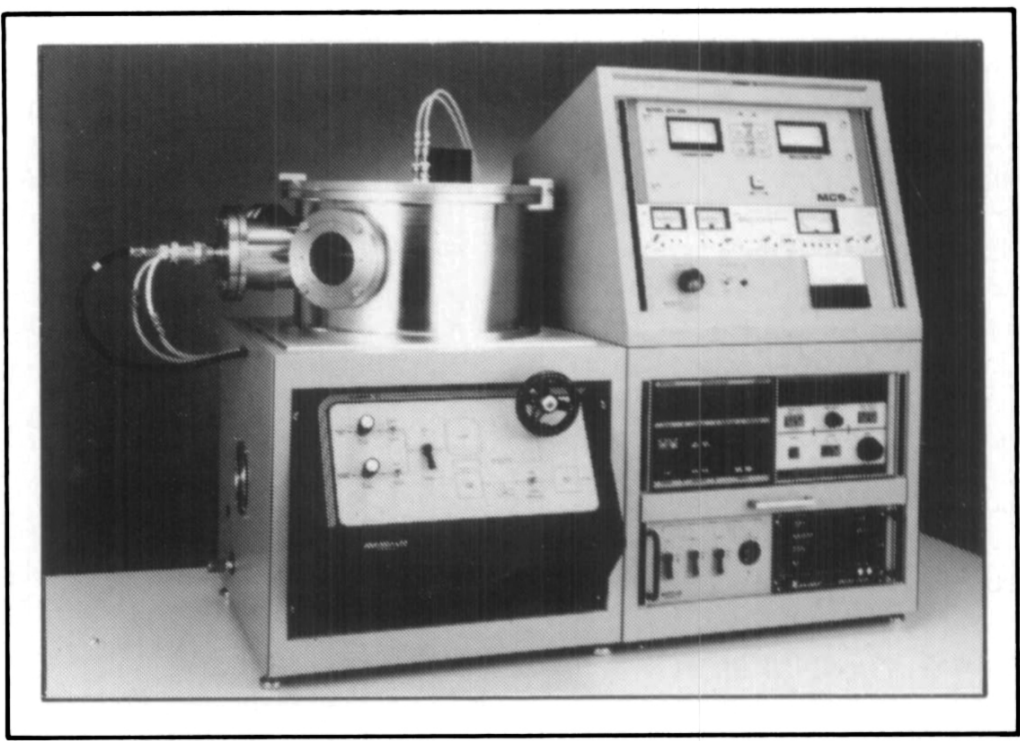

Anatech LTD offers the Research XII RFPM research sputtering system for this film deposition. The System is table top, designed for simple operation, and is capable of sputtering a broad range of materials by either RF or DC power.

The system can be configured with various options, including additional sputtering sources and power supplies, ion beam source, temperature and thickness control, heated or cooled stage, and pumping capacity.

Call or write today for techical information.

\section{ANATECH LTD \\ 5510 Vine Street, Alexandria, Virginia 22310 \\ Telephone (703) 971-9200 \\ Telex 858531 \\ Fax 703-971-4818}

